\title{
Stabilization of multidimensional behaviors
}

\author{
Paula Rocha*†
}

\begin{abstract}
We consider three types of stabilizability defined for discrete $\mathrm{nD}$ systems within the behavioral framework, namely: trajectory stabilizability, set- controllability to a stable behavior and stabilizability by interconnection. As a first step, we introduce and fully characterize the underlying stability notion. Then, we formalize the definitions of these properties and investigate what is the relationship among them.
\end{abstract}

\section{Introduction}

As is well known, the central idea in the behavioral approach to control is the one of interconnection. This consists in the intersection of a given behavior to be controlled with a suitable controller-behavior, in order to obtain a desired (controlled) behavior. The classical stabilization problem can be then reformulated in terms of

${ }^{*}$ Department of Mathematics, University of Aveiro, 3810-193 Portugal (procha@ mat.ua.pt)

${ }^{\dagger}$ This work was supported by the Fundação para a Ciência e Tecnologia (FCT) through the Unidade de Investigação Matemática e Aplicações (UIMA), University of Aveiro, Portugal. 
interconnections as the search for a controller-behavior that yields a stable controlled behavior. In this context, a behavior that admits a stabilizing controller is said to be stabilizable.

On the other hand, stabilizability has also been defined in terms of trajectory concatenation. Indeed, a $1 \mathrm{D}$ behavior $\mathfrak{B}$ is defined to be stabilizable if all its trajectories can be concatenated with trajectories that tend to zero as time goes to infinity, [1]; as we shall see this definition can be generalized to the multidimensional case.

Still at the level of trajectory concatenation, the notion of set controllability has been introduced in [2]. A behavior $\mathfrak{B}$ is said to be set-controllable to a subbehavior $\mathfrak{B}^{*} \subset \mathfrak{B}$ if its trajectories can be driven to $\mathfrak{B}^{*}$ in the sense that they can be concatenated with some trajectory in this sub-behavior. In this framework, a stabilizable behavior could be defined as one that is set-controllable to a stable sub-behavior.

This leads us to three different types of stabilization properties, which in the sequel will be referred to as stabilization by interconnection, trajectory stabilization and set stabilization, respectively. A natural question to pose is what is the relationship between all these properties. For the one-dimensional (1D) case, the results in [3], [2] and [1] allow to conclude that they are all equivalent. However the 1D techniques used in [3] and [1] do not extend to higher dimensions.

The main purpose of this contribution is to characterize and compare the aforementioned stabilization properties for $\mathrm{nD}$ behaviors defined over the discrete grid $\mathbb{Z}^{n}$. Clearly, this presupposes a definition of stability. Here we shall adapt the one introduced in [4] for the continuous case, which is defined with respect to given stability cones. 
Some results on the stabilizability of two-dimensional systems have also been obtained in [5], but with a different underlying notion of stability and without addressing the relationship with stabilization by interconnection. On the other hand, the stabilization of $\mathrm{nD}$ behaviors by interconnection has been partially studied in [4] for the continuous case, but without a connection with trajectory- or set stabilizability.

The paper is organized as follows. Section 2 contains the necessary preliminary concepts and results. In Section 3 we introduce the stability property to be used in the sequel and derive some new results that will be relevant for our study of stabilization. Section 4 is devoted to the characterization of the three stabilizability properties, as well as to the study of the relationship among these properties. Finally, some concluding remarks are presented in Section 5.

\section{Preliminaries}

In order to state more precisely the questions to be considered, we introduce some preliminary notions and results.

\subsection{Kernel behaviors}

We consider $\mathrm{nD}$ behaviors $\mathfrak{B}$ defined over $\mathbb{Z}^{n}$ that can be described by a set of linear partial difference equations, i.e.,

$$
\mathfrak{B}=\operatorname{ker} H\left(\underline{\sigma}, \underline{\sigma}^{-1}\right):=\left\{w \in \mathcal{U} \mid H\left(\underline{\sigma}, \underline{\sigma}^{-1}\right) w \equiv 0\right\}
$$

where $\mathcal{U}$ is the trajectory universe, here taken to be $\left(\mathbb{R}^{q}\right)^{\mathbb{Z}^{n}}, \underline{\sigma}=\left(\sigma_{1}, \ldots, \sigma_{n}\right)$, $\underline{\sigma}^{-1}=\left(\sigma_{1}^{-1}, \ldots, \sigma_{n}^{-1}\right)$, the $\sigma_{i}$ 's are the elementary $\mathrm{nD}$ shift operators (defined by 
$\sigma_{i} w(\underline{k})=w\left(\underline{k}+e_{i}\right)$, for $\underline{k} \in \mathbb{Z}^{n}$, where $e_{i}$ is the ith element of the canonical basis of $\left.\mathbb{R}^{n}\right)$ and $H\left(\underline{s}, \underline{s}^{-1}\right)$ is an $\mathrm{nD}$ Laurent-polynomial matrix known as representation of $\mathfrak{B}$. We shall refer to these behaviors as kernel behaviors. An important characterization of kernel behaviors is the following.

Theorem 1 [6] $\mathfrak{B} \subset\left(\mathbb{R}^{q}\right)^{\mathbb{Z}^{n}}$ is a kernel behavior if and only if it is a linear, shift-invariant (i.e, $\sigma_{i}(\mathfrak{B})=\mathfrak{B}, i=1, \ldots, n$ ) and closed (in the point-wise convergence topology) subspace of $\left(\mathbb{R}^{q}\right)^{\mathbb{Z}^{n}}$.

Instead of characterizing $\mathfrak{B}$ by means of a representation matrix $H$, it is also possible to characterize it by means of its orthogonal module $\operatorname{Mod}(\mathfrak{B})$, which consists of all the nD Laurent-polynomial rows $r\left(\underline{s}, \underline{s}^{-1}\right) \in \mathbb{R}^{1 \times q}\left[\underline{s}, \underline{s}^{-1}\right]$ such that $\mathfrak{B} \subset \operatorname{ker} r\left(\underline{\sigma}, \underline{\sigma}^{-1}\right)$, and can be shown to coincide with the $\mathbb{R}\left[\underline{s}, \underline{s}^{-1}\right]$-module $\operatorname{RM}(H)$ generated by the rows of $H$, i.e., $\operatorname{Mod}(\mathfrak{B})=\operatorname{RM}\left(H\left(\underline{s}, \underline{s}^{-1}\right)\right)$.

It turns out that sums and intersections of kernel behaviors can be formulated in terms of the corresponding modules.

Theorem 2 [7] Let $\mathfrak{B}_{1}$ and $\mathfrak{B}_{2}$ be two nD kernel behaviors. Then, $\mathfrak{B}_{1}+\mathfrak{B}_{2}$ and $\mathfrak{B}_{1} \cap B_{2}$ are also $n D$ kernel behaviors and

$$
\begin{aligned}
& \text { 1. } \operatorname{Mod}\left(\mathfrak{B}_{1}+\mathfrak{B}_{2}\right)=\operatorname{Mod}\left(\mathfrak{B}_{1}\right) \cap \operatorname{Mod}\left(\mathfrak{B}_{2}\right) \\
& \text { 2. } \operatorname{Mod}\left(\mathfrak{B}_{1} \cap \mathfrak{B}_{2}\right)=\operatorname{Mod}\left(\mathfrak{B}_{1}\right)+\operatorname{Mod}\left(\mathfrak{B}_{2}\right)
\end{aligned}
$$

The notions of controllability and autonomy play an important role in the sequel. Although controllability is originally defined in a different way, here we say that an $\mathrm{nD}$ kernel behavior $\mathfrak{B}=\operatorname{ker} R\left(\underline{\sigma}, \underline{\sigma}^{-1}\right)$ is controllable if besides being a kernel it is also an image, i.e., if there exists $M\left(\underline{s}, \underline{s}^{-1}\right)$ such that $\mathfrak{B}=\operatorname{im} M\left(\underline{\sigma}, \underline{\sigma}^{-1}\right)$. On the other hand, we say that a behavior is autonomous if it has no free variables 
(or inputs). $\mathfrak{B}=\operatorname{ker} R\left(\underline{\sigma}, \underline{\sigma}^{-1}\right)$ is autonomous if and only if $R\left(\underline{s}, \underline{s}^{-1}\right)$ has full column rank (over $\mathbb{R}\left[\underline{s}, \underline{s}^{-1}\right]$ ), [8].

As also shown in [8], every $\mathrm{nD}$ kernel behavior $\mathfrak{B}$ can be decomposed into a sum

$$
\mathfrak{B}=\mathfrak{B}^{c}+\mathfrak{B}^{a},
$$

where $\mathfrak{B}^{c}$ is the controllable part of $\mathfrak{B}$ (defined as the largest controllable subbehavior of $\mathfrak{B}$ ) and $\mathfrak{B}^{a}$ is a (non-unique) autonomous sub-behavior said to be an autonomous part of $\mathfrak{B}$. If $\mathfrak{B}^{a}$ is an autonomous part of $\mathfrak{B}$ such that $\mathfrak{B}=\mathfrak{B}^{c} \oplus \mathfrak{B}^{a}$, we shall say that $\mathfrak{B}^{a}$ is a direct summand of $\mathfrak{B}$.

An interesting case is when the controllable part $\mathfrak{B}^{c}$ is rectifiable. An $\mathrm{nD}$ behavior $\mathfrak{B}=\operatorname{ker} R\left(\underline{\sigma}, \underline{\sigma}^{-1}\right) \subset\left(\mathbb{R}^{q}\right)^{\mathbb{Z}^{n}}$ is said to be rectifiable if there exists an invertible operator $U\left(\underline{\sigma}, \underline{\sigma}^{-1}\right)$, where $U\left(\underline{s}, \underline{s}^{-1}\right)$ is an $\mathrm{nD}$ Laurent-polynomial matrix, such that $U\left(\underline{\sigma}, \underline{\sigma}^{-1}\right)(\mathfrak{B})=\operatorname{ker}\left[\begin{array}{ll}I_{l} & 0\end{array}\right]$, where $I_{l}$ is the $l \times l$ identity matrix, for some $l \in\{1, \ldots, q\}$. This is equivalent to say that $R\left(\underline{s}, \underline{s}^{-1}\right)$ is zero left-prime (ZLP), see [2]. When a rectifying operator exists, it is possible to take advantage of the simplified form of the rectified behaviors in order to derive various results. In particular, it is not difficult to obtain the next proposition.

Proposition 1 Let $\mathfrak{B}=\operatorname{ker} R\left(\underline{\sigma}, \underline{\sigma}^{-1}\right) \subset\left(\mathbb{R}^{q}\right)^{\mathbb{Z}^{n}}$ be an $n D$ kernel behavior with rectifiable controllable part $\mathfrak{B}^{c}$ and $U\left(\underline{\sigma}, \underline{\sigma}^{-1}\right)$ be a corresponding rectifying operator such that $U\left(\underline{\sigma}, \underline{\sigma}^{-1}\right)\left(\mathfrak{B}^{c}\right)=\operatorname{ker}\left[\begin{array}{ll}I_{l} & 0\end{array}\right]$. Then the following are equivalent.

1. $\mathfrak{B}=\mathfrak{B}^{c} \oplus \mathfrak{B}^{a}$

2. $\mathfrak{B}^{a}=\operatorname{ker}\left[\begin{array}{cc}P & 0 \\ X & I_{q-l}\end{array}\right] U$, with $P\left(\underline{s}, \underline{s}^{-1}\right)$ such that $R=\left[\begin{array}{ll}P & 0\end{array}\right] U$ and $X\left(\underline{s}, \underline{s}^{-1}\right)$ an arbitrary Laurent-polynomial matrix of suitable size. 
Note that the behaviors $\mathfrak{B}^{a}$ of Proposition 1 always exist and are autonomous. Thus, this result states that every behavior with rectifiable controllable part has direct summands and, moreover, gives a parametrization for all such summands. This yields the following parametrization of $U(\mathfrak{B})$.

Corollary 1 Let $\mathfrak{B}=\operatorname{ker} R\left(\underline{\sigma}, \underline{\sigma}^{-1}\right) \subset\left(\mathbb{R}^{q}\right)^{\mathbb{Z}^{n}}$ be an $n D$ kernel behavior with rectifiable controllable part $\mathfrak{B}^{c}$ and $U\left(\underline{\sigma}, \underline{\sigma}^{-1}\right)$ be a corresponding rectifying operator such that $U\left(\underline{\sigma}, \underline{\sigma}^{-1}\right)\left(\mathfrak{B}^{c}\right)=\operatorname{ker}\left[\begin{array}{ll}I_{l} & 0\end{array}\right]$. Then

$$
U(\mathfrak{B})=\left[\begin{array}{c}
0 \\
\left(\mathbb{R}^{q-l}\right)^{\mathbb{Z}^{n}}
\end{array}\right] \oplus\left[\begin{array}{l}
I_{l} \\
Y
\end{array}\right] \operatorname{ker} P,
$$

with $P\left(\underline{s}, \underline{s}^{-1}\right)$ such that $R=\left[\begin{array}{ll}P & 0\end{array}\right] U$ and $Y\left(\underline{s}, \underline{s}^{-1}\right)$ an arbitrary Laurentpolynomial matrix of suitable size.

To conclude this subsection, we present two theorems that are crucial for our purposes.

The first one is an immediate consequence of the variable elimination property obtained in [9] and states that the components of the variable of a $\mathrm{nD}$ kernel behavior are themselves trajectories of an $\mathrm{nD}$ kernel behavior.

More concretely, given a behavior $\mathfrak{B} \subset\left(\mathbb{R}^{q}\right)^{\mathbb{Z}^{n}}$, with variable $w=\left[w_{1} \ldots w_{q}\right]^{T}$, define its projection with respect to the ith-component of $w$ as

$$
\Pi_{i}(\mathfrak{B})=\left\{w_{i} \in \mathbb{R}^{\mathbb{Z}^{n}} \mid \exists w_{j}, i \neq j=1, \ldots, q:\left[w_{1} \ldots w_{q}\right]^{T} \in \mathfrak{B}\right\}
$$

Theorem 3 If $\mathfrak{B} \subset\left(\mathbb{R}^{q}\right)^{\mathbb{Z}^{n}}$ is an $n D$ kernel behavior whose variable w has components $w_{i}, i=1, \ldots, q$, then $\Pi_{i}(\mathfrak{B})$ is also an $n D$ kernel behavior.

The second important theorem is a consequence of the results and reasonings presented in [10] and amounts to say that the restriction of an $\mathrm{nD}$ kernel behavior to 
one of the axes in $\mathbb{Z}^{n}$ is a $1 \mathrm{D}$ kernel behavior.

To state this more precisely, given a kernel behavior $\mathfrak{B} \subset\left(\mathbb{R}^{q}\right)^{\mathbb{Z}^{n}}$, define its restriction to the axis $k e_{j}, k \in \mathbb{Z}$ as

$$
\mathfrak{B}_{j}=\left\{v(k) \in\left(\mathbb{R}^{q}\right)^{\mathbb{Z}} \mid \exists w \in \mathfrak{B}: w\left(k e_{j}\right)=v(k)\right\} .
$$

Moreover, consider the map $\mathcal{L}_{j}:\left(\mathbb{R}^{q}\right)^{\mathbb{Z}^{n}} \rightarrow\left(\mathbb{R}^{q}\right)^{\mathbb{Z}}$ defined by $\mathcal{L}_{j}(w)=v_{j}$, with $v_{j}(k)=w\left(k e_{j}\right), k \in \mathbb{Z}$. This map is linear, and is continuous when the vector spaces $\left(\mathbb{R}^{q}\right)^{\mathbb{Z}^{n}}$ and $\left(\mathbb{R}^{q}\right)^{\mathbb{Z}}$ are equipped with the topology of point-wise convergence. This allows to prove (cf. [10]) that $\mathfrak{B}_{j}=\mathcal{L}_{j}(\mathfrak{B})$ is a linear closed subspace of $\left(\mathbb{R}^{q}\right)^{\mathbb{Z}}$. Since, moreover, $\mathfrak{B}_{j}$ is clearly (1D) shift-invariant, by Theorem 1 we conclude that $\mathfrak{B}_{j}$ is a kernel behavior.

Theorem 4 If $\mathfrak{B} \subset\left(\mathbb{R}^{q}\right)^{\mathbb{Z}^{n}}$ is an $n D$ kernel behavior, then the behavior $\mathfrak{B}_{j}$ (as previously defined) is a 1D kernel behavior.

\subsection{Interconnections and control}

Given two behaviors $\mathfrak{B}^{1}$ and $\mathfrak{B}^{2}$ their interconnection is defined as the intersection $\mathfrak{B}^{1} \cap \mathfrak{B}^{2}$. This interconnection is said to be regular if

$$
\operatorname{Mod}\left(\mathfrak{B}^{1}\right) \cap \operatorname{Mod}\left(\mathfrak{B}^{2}\right)=\{0\}
$$

Regular interconnections correspond to a lack of overlapping between the laws of the interconnected behaviors and play an important role in behavioral control, [1]. Based on the notion of behavior interconnection it is possible to formulate a control problem in set theoretic terms. Indeed, if $\mathfrak{P}$ is the behavior of the system to be controlled (the plant) and $\mathfrak{C}$ is the set of all signals compatible with the additional 
restrictions to be imposed on $w$, i.e., the controller, then the resulting controlled behavior is given by the interconnection

$$
\mathfrak{P} \cap \mathfrak{C}
$$

of the behaviors $\mathfrak{P}$ and $\mathfrak{C}$. Thus, in the behavioral setting, a control problem consists in, given a desired controlled behavior $\mathfrak{D}$, finding a full controller $\mathfrak{C}$ such that its interconnection (1) with the plant behavior $\mathfrak{P}$ results in $\mathfrak{D}$. In case this interconnection is regular, the controller is called a regular controller and the desired behavior $\mathfrak{D}$ is said to be achievable by regular interconnection.

Achievability by regular interconnection has been characterized in [2] for general pairs of behaviors and desired sub-behaviors. Here we shall consider a particular case, namely the achievability of direct summands of a behavior by regular interconnection.

Theorem 5 Let $\mathfrak{B} \subset\left(\mathbb{R}^{q}\right)^{\mathbb{Z}^{n}}$ and $\mathfrak{B}^{*} \subset \mathfrak{B}$ be two $n D$ kernel behaviors such that $\mathfrak{B}=\mathfrak{B}^{c} \oplus \mathfrak{B}^{*}$, where $\mathfrak{B}^{c}$ denotes the controllable part of $\mathfrak{B}$. Then the following conditions are equivalent:

1. $\mathfrak{B}^{*}$ is achievable from $\mathfrak{B}$ by regular interconnection

2. $\mathfrak{B}^{c}$ is rectifiable.

\section{Proof:}

$1 \Rightarrow 2$ : The fact that $\mathfrak{B}=\mathfrak{B}^{c} \oplus \mathfrak{B}^{*}$ can be formulated in terms of modules as

$$
\operatorname{Mod}\left(\mathfrak{B}^{c}\right) \cap \operatorname{Mod}\left(\mathfrak{B}^{*}\right)=\operatorname{Mod}(\mathfrak{B})
$$

and

$$
\operatorname{Mod}\left(\mathfrak{B}^{c}\right)+\operatorname{Mod}\left(\mathfrak{B}^{*}\right)=\mathbb{R}^{1 \times q}\left[\underline{s}, \underline{s}^{-1}\right]
$$


Assume that $\mathfrak{B}^{*}$ is achievable from $\mathfrak{B}$ by regular interconnection. This means that there exists a controller behavior $\mathfrak{C}$ such that, again in terms of modules,

$$
\operatorname{Mod}(\mathfrak{B})+\operatorname{Mod}(\mathfrak{C})=\operatorname{Mod}\left(\mathfrak{B}^{*}\right)
$$

and

$$
\operatorname{Mod}(\mathfrak{B}) \cap \operatorname{Mod}(\mathfrak{C})=\{0\} \subset \mathbb{R}^{1 \times q}\left[\underline{s}, \underline{s}^{-1}\right]
$$

Now, from (3) and (4), and taking into account that $\operatorname{Mod}(\mathfrak{B}) \subset \operatorname{Mod}\left(\mathfrak{B}^{c}\right)$ (cf. (2)), it follows that

$$
\operatorname{Mod}\left(\mathfrak{B}^{c}\right)+\operatorname{Mod}(\mathfrak{C})=\mathbb{R}^{1 \times q}\left[\underline{s}, \underline{s}^{-1}\right] .
$$

On the other hand, since (by (4)) $\operatorname{Mod}(\mathfrak{C}) \subset \operatorname{Mod}\left(\mathfrak{B}^{*}\right), \operatorname{Mod}\left(\mathfrak{B}^{c}\right) \cap \operatorname{Mod}\left(\mathfrak{C}^{\mathfrak{c}}\right) \subset$ $\operatorname{Mod}\left(\mathfrak{B}^{c}\right) \cap \operatorname{Mod}\left(\mathfrak{B}^{*}\right)=\operatorname{Mod}(\mathfrak{B})$. This clearly implies that $\operatorname{Mod}\left(\mathfrak{B}^{c}\right) \cap \operatorname{Mod}(\mathfrak{C}) \subset$ $\operatorname{Mod}(\mathfrak{B}) \cap \operatorname{Mod}(\mathfrak{C})$ and therefore, due to (5),

$$
\operatorname{Mod}\left(\mathfrak{B}^{c}\right) \cap \operatorname{Mod}(\mathfrak{C})=\{0\} \subset \mathbb{R}^{1 \times q}\left[\underline{s}, \underline{s}^{-1}\right]
$$

Equations (6) and (7) mean that the zero behavior $\{0\} \subset\left(\mathbb{R}^{q}\right)^{\mathbb{Z}^{n}}$ is achievable from $\mathfrak{B}^{c}$ by regular interconnection, i.e., $\mathfrak{B}^{c}$ is rectifiable.

$2 \Rightarrow 1$ : Assume now that $\mathfrak{B}^{c}$ is rectifiable. Let $U\left(\underline{\sigma}, \underline{\sigma}^{-1}\right)$ be an invertible operator such that

$$
U\left(\underline{\sigma}, \underline{\sigma}^{-1}\right)\left(\mathfrak{B}^{c}\right)=\operatorname{ker}\left[\begin{array}{ll}
I_{l} & 0
\end{array}\right]
$$

Then the achievability of $\mathfrak{B}^{*}$ from $\mathfrak{B}$ by regular interconnection is equivalent to the achievability of $U\left(\underline{\sigma}, \underline{\sigma}^{-1}\right)\left(\mathfrak{B}^{*}\right)$ from $U\left(\underline{\sigma}, \underline{\sigma}^{-1}\right)(\mathfrak{B})$ by regular interconnection. Moreover, note that, since $\mathfrak{B}=\mathfrak{B}^{c} \oplus \mathfrak{B}^{*}$, also

$$
U\left(\underline{\sigma}, \underline{\sigma}^{-1}\right)(\mathfrak{B})=U\left(\underline{\sigma}, \underline{\sigma}^{-1}\right)\left(\mathfrak{B}^{c}\right) \oplus U\left(\underline{\sigma}, \underline{\sigma}^{-1}\right)\left(\mathfrak{B}^{*}\right) .
$$


This implies that, for some nD Laurent-polynomial matrices $P\left(\underline{s}, \underline{s}^{-1}\right)$ and $X\left(\underline{s}, \underline{s}^{-1}\right)$ with $l$ columns,

$$
U\left(\underline{\sigma}, \underline{\sigma}^{-1}\right)\left(\mathfrak{B}^{*}\right)=\operatorname{ker}\left[\begin{array}{cc}
P\left(\underline{\sigma}, \underline{\sigma}^{-1}\right) & 0 \\
X\left(\underline{\sigma}, \underline{\sigma}^{-1}\right) & I_{q-l}
\end{array}\right]
$$

and

$$
U\left(\underline{\sigma}, \underline{\sigma}^{-1}\right)(\mathfrak{B})=\operatorname{ker}\left[P\left(\underline{\sigma}, \underline{\sigma}^{-1}\right) 0\right] .
$$

Thus, $U\left(\underline{\sigma}, \underline{\sigma}^{-1}\right)\left(\mathfrak{B}^{*}\right)$ is achievable by the interconnection of $\operatorname{ker}\left[X\left(\underline{\sigma}, \underline{\sigma}^{-1}\right) I_{q-l}\right]$ with $U\left(\underline{\sigma}, \underline{\sigma}^{-1}\right)(\mathfrak{B})$, which is a regular one, allowing to conclude that also the behavior $\mathfrak{B}^{*}$ is achievable from $\mathfrak{B}$ by regular interconnection.

\subsection{Set-controllability}

Another notion that will be relevant in our study is the one of set-controllability. This is defined in terms of trajectory concatenability. Two trajectories $w_{1}$ and $w_{2}$ of a behavior $\mathfrak{B}$ evolving over $\mathbb{R}^{n}$ are said to be concatenable with respect to a pair $\left(T_{1}, T_{2}\right)$ of subsets of $\mathbb{R}^{n}$ if there exists a third trajectory $w \in \mathfrak{B}$ coinciding with $w_{1}$ in $T_{1}$ and with $w_{2}$ in $T_{2}$.

Definition 1 An $n D$ behavior $\mathfrak{B}$ is said to be set-controllable to a sub-behavior $\mathfrak{B}^{*} \subset \mathfrak{B}$ if the following holds: there exists a $\rho>0$ such that for any $w \in \mathfrak{B}$ there exists $w^{*} \in \mathfrak{B}^{*}$ such that, for any sets $T_{1}, T_{2} \subset \mathbb{Z}^{n}$ with $d\left(T_{1}, T_{2}\right) \geq \rho$, w and $w^{*}$ are concatenable with respect to $\left(T_{1}, T_{2}\right)$.

Here, $w$ can be interpreted as a given system trajectory and $w^{*}$ is some trajectory in the desired subsystem into which $w$ can be controlled by means of an adequate driving trajectory. Among others, the following characterization of setcontrollability is given in [2]. 
Theorem 6 Let $\mathfrak{B}$ and $\mathfrak{B}^{*} \subset \mathfrak{B}$ be two $n D$ kernel behaviors. Then $\mathfrak{B}$ is setcontrollable to $\mathfrak{B}^{*}$ if and only if

$$
\mathfrak{B}=\mathfrak{B}^{c}+\mathfrak{B}^{*},
$$

where $\mathfrak{B}^{c}$ denotes the controllable behavior of $\mathfrak{B}$.

The connection between set-controllability to a behavior $\mathfrak{B}^{*} \subset \mathfrak{B}$ and achievability of $\mathfrak{B}^{*}$ (from $\mathfrak{B}$ ) by regular interconnection has been investigated in [2].

Theorem 7 [2] Let $\mathfrak{B}$ and $\mathfrak{B}^{*} \subset \mathfrak{B}$ be two $n D$ kernel behaviors. If $\mathfrak{B}^{*}$ is achievable from $\mathfrak{B}$ by regular interconnection then $\mathfrak{B}$ is set-controllable to $\mathfrak{B}^{*}$.

However, for $n \geq 2$ set-controllability does not imply achievability by regular interconnection.

\section{Stability}

A discrete $1 \mathrm{D}$ behavior $\mathfrak{B} \subset\left(\mathbb{R}^{q}\right)^{\mathbb{Z}}$ is said to be stable if all its trajectories tend to the origin as time goes to infinity. In the $\mathrm{nD}$ case, we shall define stability with respect to a specified stability region by adapting the ideas in [4] to the discrete case. For this purpose we identify a direction in $\mathbb{Z}^{n}$ with an element $\underline{d}=\left(d_{1}, \ldots, d_{n}\right) \in \mathbb{Z}^{n}$ whose components are coprime integers, and define a stability cone in $\mathbb{Z}^{n}$ as the set of all positive integer linear combinations of $n$ linearly independent directions. This is formalized as follows.

Definition $2 A$ stability cone $S \subset \mathbb{Z}^{n}$ is a set of the form

$$
S=\left\{s=\alpha_{1} \underline{d}_{1}+\ldots+\alpha_{n} \underline{d}_{n} \mid \alpha_{i} \in \mathbb{Z}_{0}^{+}, i=1, \ldots n\right\}
$$

where $\underline{d}_{1}, \ldots \underline{d}_{n}$ are $n$ linearly independent directions in $\mathbb{Z}^{n}$. 
Note that, according to this definition, a stability cone is always a "full" cone, in the sense that it contains a basis of directions.

By a half-line associated with a direction $\underline{d} \in \mathbb{Z}^{n}$ we mean the set of all points of the form $\alpha \underline{d}$ where $\alpha$ is a nonnegative integer; clearly, the half-lines in a stability cone $S$ are the ones associated with the directions $\underline{d} \in S$.

Definition 3 Given a stability cone $S \subset \mathbb{Z}^{n}$, a trajectory $w \in\left(\mathbb{R}^{q}\right)^{\mathbb{Z}^{n}}$ is said to be $S$-stable if it tends to zero along every half line in $S . A$ behavior $\mathfrak{B}$ is $S$-stable if all its trajectories are $S$-stable.

Lemma 1 Every $n D$ kernel behavior $\mathfrak{B} \subset\left(\mathbb{R}^{q}\right)^{\mathbb{Z}^{n}}$ which is stable with respect to some stability cone $S$ is a finite dimensional linear subspace of the trajectory universe, $\left(\mathbb{R}^{q}\right)^{\mathbb{Z}^{n}}$.

Proof: Assume that the behavior $\mathfrak{B}$ is $S$-stable, for some stability cone $S$. It is not difficult to see that an appropriate change of independent variables transforms $S$ into the first orthant. Therefore we shall assume, without loss of generality, that $S$ is the first orthant in $\mathbb{Z}^{n}$.

Let then $\mathfrak{B} \subset\left(\mathbb{R}^{q}\right)^{\mathbb{Z}^{n}}$ be an $n \mathrm{D}$ behavior which is stable with respect to the first orthant. According to the definition of stability, this means that the system trajectories $w$ tend to zero along the positive axes defined by $e_{1}, \ldots, e_{n}$, i.e., $\lim _{k \rightarrow+\infty} w\left(k e_{j}\right)=0$, for $j=1, \ldots, n$. This implies that the same happens for the components $w_{i}$ of $w, i=1, \ldots, q$. In other words, each univariate $n \mathrm{D}$ behavior $\Pi_{i} \mathfrak{B}$ is stable with respect to the first orthant.

Consider now the restriction $\left(\Pi_{i} \mathfrak{B}\right)_{j}=\mathcal{L}_{j}\left(\Pi_{i} \mathfrak{B}\right)$ of the behavior $\Pi_{i} \mathfrak{B}$ to the axis $k e_{j}, k \in \mathbb{Z}$. By Theorem 4 , this is a (univariate) 1D kernel behavior. Moreover, $\left(\Pi_{i} \mathfrak{B}\right)_{j}$ is stable, since its trajectories $v_{j}$ clearly satisfy $\lim _{k \rightarrow+\infty} v_{j}(k)=0$. 
This implies that the variable $v_{j}$ is not free, which in turn means that $\left(\Pi_{i} \mathfrak{B}\right)_{j}=$ $\operatorname{ker} p_{j}\left(\sigma, \sigma^{-1}\right)$ for some nonzero Laurent-polynomial $p_{j}\left(s, s^{-1}\right)$, where $\sigma$ denotes the 1D shift. Translating this in terms of the trajectories $w_{i}$, we obtain

$$
p_{j}\left(\sigma_{j}, \sigma_{j}^{-1}\right) w_{i}\left(k e_{j}\right)=0, k \in \mathbb{Z},
$$

and, by the shift-invariance of $\mathfrak{B}$, we may conclude that

$$
p_{j}\left(\sigma_{j}, \sigma_{j}^{-1}\right) w_{i} \equiv 0 .
$$

Letting $j=1, \ldots, n$, yields that the components $w_{i}$ of the system trajectories $w \in \mathfrak{B}$ must satisfy the following set of $n$ decoupled difference equations:

$$
\left[\begin{array}{c}
p_{1}\left(\sigma_{1}, \sigma_{1}^{-1}\right) \\
\vdots \\
p_{n}\left(\sigma_{n}, \sigma_{n}^{-1}\right)
\end{array}\right] w_{i} \equiv\left[\begin{array}{c}
0 \\
\vdots \\
0
\end{array}\right] .
$$

It is not difficult to check that, under these conditions, $\mathfrak{B}_{i}$ is a finite dimensional subspace of $\mathbb{R}^{\mathbb{Z}^{n}}, i=1, \ldots, q$.

Since $\mathfrak{B} \subset \mathfrak{B}_{1} \times \ldots \times \mathfrak{B}_{q}$, we conclude that also $\mathfrak{B}$ is finite dimensional.

Finite dimensional $n \mathrm{D}$ behaviors are known as strongly autonomous [4]. Thus, stability with respect to a stability cone $S$ implies strong autonomy, leaving out the class of infinite dimensional autonomous behaviors. The definition of stability used in [5] does not exclude this class of systems, but is focussed on 2D behaviors and seems to be somewhat difficult to generalize to the higher dimensional case. In order to characterize stability, it is convenient to introduce some preliminary notation. Given two elements $\underline{\lambda}=\left(\lambda_{1}, \ldots, \lambda_{n}\right) \in \mathbb{C}^{n}$ and $\underline{k}=\left(k_{1}, \ldots, k_{n}\right) \in \mathbb{Z}^{n}$, we define

$$
\underline{\lambda}^{\underline{k}}:=\lambda_{1}^{k_{1}} \ldots \lambda_{n}^{k_{n}} .
$$


With this notation an $\mathrm{nD} q$-vector polynomial function $p(\underline{k})$ of $\underline{k}$ is such that

$$
p(\underline{k})=\sum_{\underline{i} \in I} \alpha_{\underline{i}} \underline{k}^{\underline{i}},
$$

where $I \subset\left(\mathbb{Z}_{+}\right)^{n}$ is a finite multi-index set and $\alpha_{\underline{i}} \in \mathbb{R}^{q}$.

Given an nD Laurent-polynomial matrix $R\left(\underline{s}, \underline{s}^{-1}\right)$ with full column rank, a zero of $R$ is defined as $\underline{\lambda} \in(\mathbb{C} \backslash\{0\})^{n}$ such that

$$
\operatorname{rank} R\left(\underline{\lambda}, \underline{\lambda}^{-1}\right)<\operatorname{rank} R\left(\underline{s}, \underline{s}^{-1}\right)
$$

where, the first rank is taken over $\mathbb{C}$ and the second one over $\mathbb{R}\left[\underline{s}, \underline{s}^{-1}\right]$. Now, let $\mathfrak{B}$ be an autonomous behavior. Since the set of zeros of the different representations of $\mathfrak{B}$ coincide, we may define the set $\mathcal{N}(\mathfrak{B})$ of zeros of the behavior $\mathfrak{B}$ as the set of zeros of any of its kernel representations. As pointed out in [8], $\mathfrak{B}$ is finite dimensional if and only of $\mathcal{N}(\mathfrak{B})$ is a finite set. In the sequel, whenever we refer to $\mathcal{N}(\mathfrak{B})$ we implicitly suppose that $\mathfrak{B}$ is autonomous.

Theorem 8 Let $\mathfrak{B} \subset\left(\mathbb{R}^{q}\right)^{\mathbb{Z}^{n}}$ be an $n D$ kernel behavior, and let $S$ be a stability cone. The the following are equivalent:

1. $\mathfrak{B}$ is S-stable

2. $\mathcal{N}(\mathfrak{B})$ is finite and for every zero $\underline{\lambda} \in \mathcal{N}(\mathfrak{B})$ and every direction $\underline{d} \in S$,

$$
|\underline{\underline{\lambda}} \underline{\underline{d}}|<1
$$




\section{Proof:}

$1 \Rightarrow 2$ : Assume that $\mathfrak{B}$ is an $S$-stable behavior. Then, by Lemma $1, \mathfrak{B}$ is finite dimensional and hence $\mathcal{N}(\mathfrak{B})$ is finite. Now, for each $\underline{\lambda} \in \mathcal{N}(\mathfrak{B})$, there exists a vector $v_{\underline{\lambda}} \in \mathbb{R}^{q} \backslash\{0\}$ such that the trajectory $w_{\underline{\lambda}}$ defined by $w_{\underline{\lambda}}(\underline{k})=v_{\underline{\lambda}} \underline{\lambda}^{\underline{k}}$ is in $\mathfrak{B}$ (here, for simplicity, we identify $\mathfrak{B}$ with its complexification, since the stability properties of these two behaviors are the same). Thus, $w_{\underline{\lambda}}$ must tend to zero along the half-line $k \underline{d}, k \in \mathbb{Z}, k \geq 0$, associated with every direction $\underline{d} \in S$. But $w_{\underline{\lambda}}(k \underline{d})=v_{\underline{\lambda}}\left(\underline{\lambda}^{\underline{d}}\right)^{k}$, and therefore the requirement that $\lim _{k \rightarrow+\infty} w_{\underline{\lambda}}(k \underline{d})=0$ implies that $\left|\underline{\lambda}^{\underline{d}}\right|<1$.

$2 \Rightarrow 1:$ Assume now that $\mathcal{N}(\mathfrak{B})$ is finite. Then, every trajectory $w \in \mathfrak{B}$ is a linear combination of trajectories of the form $w_{(p, \underline{\lambda})}(\underline{k})=p(\underline{k}) \underline{\lambda}^{\underline{k}}$, for some suitable nonzero $\mathrm{nD} q$-vector polynomial function $p(\underline{k})$. Since, for $k \in \mathbb{Z}, w_{(p, \underline{\lambda})}(k \underline{d})=$ $\tilde{p}(k)\left(\underline{\lambda}^{\underline{d}}\right)^{k}$, for some 1D $q$-vector polynomial function $\tilde{p}(k)$, if $\left|\underline{\lambda}^{\underline{d}}\right|<1$ for every direction $\underline{d} \in S, \lim _{k \rightarrow+\infty} w_{(p, \underline{\lambda})}(k \underline{d})=0$ and, consequently the same happens for $w$. Thus $\mathfrak{B}$ is $S$-stable.

Motivated by this result, given a stability cone $S$, we shall say that $\underline{\lambda} \in \mathbb{C}^{n}$ is $S$-stable if for every direction $\underline{d} \in S,|\underline{\lambda} \underline{d}|<1$.

Thus, Theorem 8 can be rephrased as: $\mathfrak{B}$ is $S$-stable if and only if it has a finite number of zeros and these zeros are $S$-stable. The $S$-stability of the zeros of $\mathfrak{B}$ is the discrete version of the sufficient condition obtained in [4] for the continuous case. The fact that in our case this a necessary and sufficient condition is a consequence of the finite dimensionality of discrete $S$-stable behaviors. 


\section{Stabilization properties}

\subsection{Stabilization by interconnection}

Our definition of $S$-stabilizability by interconnection is similar to the definition of stabilizability with respect to a cone $S$ proposed in [4], but has the extra requirement of regularity.

Definition 4 Given a stability cone $S \subset \mathbb{Z}^{n}$, we say that an $n D$ kernel behavior $\mathfrak{B} \subset\left(\mathbb{R}^{q}\right)^{\mathbb{Z}^{n}}$ is $S$-stabilizable by interconnection if there exists an $S$-stable subbehavior $\mathfrak{B}^{s} \subset \mathfrak{B}$ that is achievable from $\mathfrak{B}$ by regular interconnection.

An easy consequence of Theorems 7 and 6 is the following necessary condition.

Theorem 9 Let $\mathfrak{B} \subset\left(\mathbb{R}^{q}\right)^{\mathbb{Z}^{n}}$ be an $n D$ kernel behavior and $S \subset \mathbb{Z}^{n}$ be a stability cone. Let further $\mathfrak{B}^{c}$ denote the controllable part of $\mathfrak{B}$. If $\mathfrak{B}$ is $S$-stabilizable by interconnection then there exists an $S$-stable sub-behavior $\mathfrak{B}^{s}$ of $\mathfrak{B}$ such that $\mathfrak{B}=\mathfrak{B}^{c}+\mathfrak{B}^{s}$.

The condition of the theorem is not sufficient since the stable summand $\mathfrak{B}^{s}$ is not guaranteed to be achievable from $\mathfrak{B}$ by regular interconnection. If $\mathfrak{B}^{s}$ happens to be a direct summand of $\mathfrak{B}$, then, by Theorem 5 , it is achievable from $\mathfrak{B}$ by regular interconnection if and only if $\mathfrak{B}^{c}$ is rectifiable.

On the other hand, if we restrict ourselves to the rectifiable case it is not difficult to prove that $\mathfrak{B}$ is $S$-stabilizable by interconnection if and only if there exists a stable behavior $\mathfrak{B}^{s}$ which is a direct summand of $\mathfrak{B}$. Recalling Proposition 1 , and using the therein defined notation, if $U$ is an operator that rectifies $\mathfrak{B}^{c}$, we obtain 
that

$$
U\left(\mathfrak{B}^{s}\right)=\operatorname{ker}\left[\begin{array}{cc}
P & 0 \\
X & I_{q-l}
\end{array}\right] .
$$

Therefore, $U\left(\mathfrak{B}^{s}\right)$ is $S$-stable if and only if ker $P$ is $S$-stable. This clearly applies to any other direct summand of $\mathfrak{B}$. Moreover $U\left(\mathfrak{B}^{s}\right)$ is $S$-stable if and only if the same holds for $\mathfrak{B}^{s}$. This allows to formulate the next characterization of $S$ stabilizability by interconnection in the rectifiable case.

Proposition 2 Let $\mathfrak{B} \subset\left(\mathbb{R}^{q}\right)^{\mathbb{Z}^{n}}=\operatorname{ker} R\left(\underline{\sigma}, \underline{\sigma}^{-1}\right)$ be an $n D$ kernel behavior and $S \subset \mathbb{Z}^{n}$ be a stability cone. Assume that the controllable part $\mathfrak{B}^{c}$ of $\mathfrak{B}$ is rectifiable. Further, let $U\left(\underline{s}, \underline{s}^{-1}\right)$ be a rectifying operator for $\mathfrak{B}^{c}$ and $P\left(\underline{s}, \underline{s}^{-1}\right)$ be such that $R=\left[\begin{array}{ll}P & 0\end{array}\right] U$. Then the following statements are equivalent.

1. $\mathfrak{B}$ is $S$-stabilizable by interconnection

2. Every direct summand of $\mathfrak{B}$ is stable

3. $\operatorname{ker} P\left(\underline{\sigma}, \underline{\sigma}^{-1}\right)$ is S-stable.

Remark Imposing the assumption of rectifiability to the controllable part of $\mathfrak{B}$ may be restrictive in the context of stabilization. However, the situation is different if instead of stabilization by interconnection one considers the more demanding problem of achieving (by regular interconnection) a stable controlled behavior $\mathfrak{B}^{s}$ which does not intersect $\mathfrak{B}^{c}$. Indeed, the solvability of this problem implies that $\mathfrak{B}=\mathfrak{B}^{c} \oplus \mathfrak{B}^{s}$, meaning that $\mathfrak{B}^{s}$ is a finite dimensional direct summand of $\mathfrak{B}$ which is achievable by regular interconnection, and hence (by Theorem 5) $\mathfrak{B}^{c}$ must be rectifiable. This observation may be relevant, for instance, in the context of pole placement. 


\subsection{Trajectory- and set stabilizability}

We shall adopt here the following definition of trajectory stabilizability.

Definition 5 Given a stability cone $S \subset \mathbb{Z}^{n}$, a behavior $\mathfrak{B} \in\left(\mathbb{R}^{q}\right)^{\mathbb{Z}^{n}}$ is said to be trajectory $S$-stabilizable if there exists a $\rho>0$ such that for any $w \in \mathfrak{B}$ there exists an $S$-stable trajectory $w^{s} \in \mathfrak{B}$ such that, for any sets $T_{1}, T_{2} \subset \mathbb{Z}^{n}$ with $d\left(T_{1}, T_{2}\right) \geq \rho, w$ and $w^{s}$ are concatenable with respect to $\left(T_{1}, T_{2}\right)$.

Note that, in particular, every trajectory in an $S$-stabilizable behavior can be "forced" to tend to zero in $S$. In this sense, our definition of trajectory stabilizability generalizes the one given in [1] for the 1D case, accordind to which a behavior $\mathfrak{B}$ is said to be stabilizable if every trajectory $w \in \mathfrak{B}$ is concatenable with a trajectory $w^{*} \in \mathfrak{B}$, such $\lim _{k \rightarrow+\infty} w^{*}(k)=0$, with respect to $\left(T_{1}, T_{2}\right)=$ $\left(\left(-\infty, k_{1}\right) \cap \mathbb{Z},\left(k_{2},+\infty\right) \cap \mathbb{Z}\right)$, for $k_{2}$ sufficiently larger than $k_{1}$.

As expected, every controllable behavior is trajectory $S$-stabilizable with respect to an arbitrary stability cone $S$, due to the fact that its trajectories are concatenable with the zero trajectory.

Trajectory stabilizability seems difficult to characterize for general behaviors. However, the problem becomes much simpler for behaviors with rectifiable controllable part. Indeed, assume that $\mathfrak{B}=\operatorname{ker} R\left(\underline{\sigma}, \underline{\sigma}^{-1}\right)$ has a rectifiable controllable part, $U\left(\underline{\sigma}, \underline{\sigma}^{-1}\right)$ is a corresponding rectifying operator, and let $P$ be such that $R=\left[\begin{array}{ll}P & 0\end{array}\right] U$. Then it is not difficult to check that $U(\mathfrak{B})$ is trajectory $S$ stabilizable if and only if ker $P\left(\underline{\sigma}, \underline{\sigma}^{-1}\right)$ is $S$-stable. Since $\mathcal{N}(\operatorname{ker} P)=\mathcal{N}\left(\mathfrak{B}^{a}\right)$ for every direct summand $\mathfrak{B}^{a}$ of $\mathfrak{B}$, this leads to the following result.

Proposition 3 Let $\mathfrak{B} \subset\left(\mathbb{R}^{q}\right)^{\mathbb{Z}^{n}}=\operatorname{ker} R\left(\underline{\sigma}, \underline{\sigma}^{-1}\right)$ be an $n D$ kernel behavior and $S \subset \mathbb{Z}^{n}$ be a stability cone. Assume that the controllable part $\mathfrak{B}^{c}$ of $\mathfrak{B}$ is recti- 
fiable. Further, let $U\left(\underline{s}, \underline{s}^{-1}\right)$ be a rectifying operator and $P\left(\underline{s}, \underline{s}^{-1}\right)$ be such that $R=\left[\begin{array}{ll}P & 0\end{array}\right] U$. Then the following statements are equivalent.

1. $\mathfrak{B}$ is trajectory $S$-stabilizable

2. Every direct summand of $\mathfrak{B}$ is stable

3. $\operatorname{ker} P\left(\underline{\sigma}, \underline{\sigma}^{-1}\right)$ is S-stable.

The concept of set stabilizabillity is formalized in the next definition.

Definition 6 Given a stability cone $S \subset \mathbb{Z}^{n}$, a behavior $\mathfrak{B} \in \mathcal{C}^{\infty}\left(\mathbb{R}^{n}, \mathbb{R}^{q}\right)$ is said to be set $S$-stabilizable if it is set-controllable to an $S$-stable sub-behavior of $\mathfrak{B}$.

An immediate consequence of Theorem 6 is the following.

Corollary 2 Given stability cone $S$, an $n D$ kernel behavior $\mathfrak{B}$, with controllable part $\mathfrak{B}^{c}$, is set $S$-stabilizable if and only if there exists an $S$-stable sub-behavior $\mathfrak{B}^{s}$ of $\mathfrak{B}$ such that

$$
\mathfrak{B}=\mathfrak{B}^{c}+\mathfrak{B}^{s}
$$

Note that, in [5], a behavior $\mathfrak{B}$ is defined to be stabilizable if $\mathfrak{B}=\mathfrak{B}^{c}+\mathfrak{B}^{s}$ with $\mathfrak{B}^{s}$ stable. Taking the previous corollary into account, and disregarding the fact that we use a different underlying stability notion, this means that the stabilizability property of [5] corresponds to set stabilizability.

Clearly, controllable behaviors are set $S$-stabilizable with respect to every stability cone $S$ as they are set-controllable to the zero behavior.

If $\mathfrak{B}^{c}$ is rectifiable, it is not difficult to show that the stability of one autonomous part $\mathfrak{B}^{s}$ implies the existence of a stable direct summand. Indeed, assume that $\mathfrak{B}^{c}$ 
is rectifiable. Then $\mathfrak{B}$ has a direct summand $\mathfrak{B}^{a}$, which implies that $\mathfrak{B}^{s} \cap \mathfrak{B}^{a}$ is also a direct summand of $\mathfrak{B}$. Moreover, this direct summand is stable since it is contained in the stable summand $\mathfrak{B}^{s}$. Now, as in the previous cases, the existence of a stable direct summand is in turn equivalent to the stability of all the direct summands of $\mathfrak{B}$. Thus, in the rectifiable case, Corollary 2 leads to a characterization of set stabilizability which is similar to the ones of trajectory stabilizability and of stabilizability by interconnection.

Proposition 4 Let $\mathfrak{B} \subset\left(\mathbb{R}^{q}\right)^{\mathbb{Z}^{n}}=\operatorname{ker} R\left(\underline{\sigma}, \underline{\sigma}^{-1}\right)$ be an $n D$ kernel behavior and $S \subset \mathbb{Z}^{n}$ be a stability cone. Assume that the controllable part $\mathfrak{B}^{c}$ of $\mathfrak{B}$ is rectifiable. Further, let $U\left(\underline{s}, \underline{s}^{-1}\right)$ be a rectifying operator and $P\left(\underline{s}, \underline{s}^{-1}\right)$ be such that $R=\left[\begin{array}{ll}P & 0\end{array}\right] U$. Then the following statements are equivalent.

1. $\mathfrak{B}$ is set $S$-stabilizable

2. Every direct summand of $\mathfrak{B}$ is stable

3. $\operatorname{ker} P\left(\underline{\sigma}, \underline{\sigma}^{-1}\right)$ is S-stable.

\subsection{Property comparison}

Clearly, set stabilizability implies trajectory stabilizability. However, the reciprocal implication is not a priori clear, since although trajectory stabilizability means that every system trajectory can be driven to a stable one, it is not a priori guarantee that all the trajectories in $\mathfrak{B}$ can be driven into a stable sub-behavior.

Combining Theorem 9 and Corollary 2, the following relation between set $S$ stabilization and $S$-stabilization by interconnection is obtained. 
Theorem 10 Let $\mathfrak{B}$ be a $n D$ kernel behavior and $S \subset \mathbb{Z}^{n}$ be a stability cone. If $\mathfrak{B}$ is $S$-stabilizable by interconnection then $\mathfrak{B}$ is set $S$-stabilizable.

The reciprocal is not true, as shown by the next example.

Example 1 Let $\mathfrak{B}=\operatorname{ker} R\left(\sigma_{1}, \sigma_{2}\right)$ be $2 D$ kernel behavior with $R\left(s_{1}, s_{2}\right)=\left[s_{1}-\right.$ $\left.1-\left(s_{2}-1\right)\right]$. Let further $S$ be the first quadrant in $\mathbb{Z}^{2}$.

It turns out that $\mathfrak{B}=\operatorname{im} M\left(\left(\sigma_{1}, \sigma_{2}\right)\right)$, with $M\left(s_{1}, s_{2}\right)=\left[\begin{array}{ll}s_{2}-1 & s_{1}-1\end{array}\right]^{T}$ and is hence controllable. By Theorem 6 , this means that $\mathfrak{B}$ is set-controllable to the zero behavior. Consequently, $\mathfrak{B}$ is set $S$-stabilizable with respect to any given stability cone.

However, $\mathfrak{B}$ is not $S$-stabilizable by interconnection. Indeed, if this were the case, there would exists a finite dimensional (S-stable) sub-behavior of $\mathfrak{B}$ which would be achievable from $\mathfrak{B}$ by regular interconnection. We next show that this is impossible.

Indeed, the S-stabilization of $\mathfrak{B}$ by interconnection is equivalent to the existence of a matrix $R^{k}\left(s_{1}, s_{2}\right)$ such that, letting $\tilde{R}^{s}=\left[\begin{array}{c}R \\ R^{k}\end{array}\right]$, $\operatorname{ker} \tilde{R}^{s}\left(\sigma_{1}, \sigma_{2}\right)$ is S-stable and, moreover, $\operatorname{RM}\left(R\left(\left(s_{1}, s_{2}\right)\right)\right) \cap \operatorname{RM}\left(R^{k}\left(\left(s_{1}, s_{2}\right)\right)\right)=\left[\begin{array}{ll}0 & 0\end{array}\right]$. This last condition implies that $R^{k}\left(s_{1}, s_{2}\right)$ must be of the form:

$$
R^{k}\left(s_{1}, s_{2}\right)=\left[\begin{array}{c}
r_{1}\left(s_{1}, s_{2}\right) \\
\vdots \\
r_{l}\left(s_{1}, s_{2}\right)
\end{array}\right]\left[\alpha\left(s_{1}, s_{2}\right) \beta\left(s_{1}, s_{2}\right)\right]
$$

for some suitable polynomials $r_{i}\left(s_{1}, s_{2}\right), i=1, \ldots, l$, and $\left(s_{2}-1\right) \alpha\left(s_{1}, s_{2}\right)+$ 
$\left(s_{1}-1\right) \beta\left(s_{1}, s_{2}\right) \neq 0$. Thus,

$$
\tilde{R}^{s}\left(s_{1}, s_{2}\right)=\left[\begin{array}{cc}
\left(s_{1}-1\right) & -\left(s_{2}-1\right) \\
r_{1}\left(s_{1}, s_{2}\right) \alpha\left(s_{1}, s_{2}\right) & r_{1}\left(s_{1}, s_{2}\right) \beta\left(s_{1}, s_{2}\right) \\
\vdots & \vdots \\
r_{l}\left(s_{1}, s_{2}\right) \alpha\left(s_{1}, s_{2}\right) & r_{l}\left(s_{1}, s_{2}\right) \beta\left(s_{1}, s_{2}\right)
\end{array}\right] .
$$

Therefore, all the nonzero maximal order minors of $\tilde{R}^{s}\left(s_{1}, s_{2}\right)$ have $p\left(s_{1}, s_{2}\right)=$ $\left(s_{2}-1\right) \alpha\left(s_{1}, s_{2}\right)+\left(s_{1}-1\right) \beta\left(s_{1}, s_{2}\right)$ as a common divisor and the zeros os this polynomial are zeros of $\operatorname{ker} \tilde{R}^{s}\left(\sigma_{1}, \sigma_{2}\right)$. But $p\left(s_{1}, s_{2}\right)$ has an infinite number os zeros, since is not a unit in $\mathbb{R}\left[s_{1}, s_{2}, s_{1}^{-1}, s_{2}^{-1}\right]$ (as $\left.p(1,1)=0\right)$. This implies that $\tilde{R}^{s}\left(s_{1}, s_{2}\right)$ has also an infinite number of zeros. Therefore its kernel is not finite dimensional and hence cannot be S-stable. This means that there exists no matrix $R^{k}\left(s_{1}, s_{2}\right)$ satisfying the desired conditions and $\mathfrak{B}$ is not $S$-stabilizable by interconnection.

Remark: Theorem 7 of [4] (adapted to the discrete case) states that an $\mathrm{nD}$ controllable behavior can always be interconnected with a suitable controller so as to obtain an $S$-stable part. This example also shows that this no longer holds when the regularity of interconnection is required.

Our conclusions on the comparison of the three considered stabilization properties can be summarized in the following diagram.

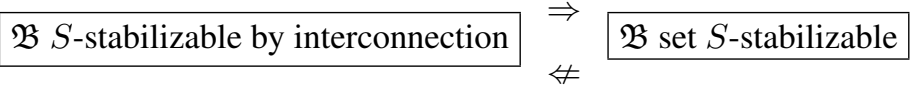

$$
\begin{aligned}
& \Downarrow \\
& \mathfrak{B} \text { is trajectory } S \text {-stabilizable }
\end{aligned}
$$

For behaviors with rectifiable controllable part, Propositions 2, 3 and 4 imply that 
all three properties are equivalent.

Theorem 11 Let $\mathfrak{B} \subset\left(\mathbb{R}^{q}\right)^{\mathbb{Z}^{n}}=\operatorname{ker} R\left(\underline{\sigma}, \underline{\sigma}^{-1}\right)$ be an $n D$ kernel behavior with rectifiable controllable part, and let $S \subset \mathbb{Z}^{n}$ be a stability cone. Then the following are equivalent:

1. $\mathfrak{B}$ is trajectory $S$-stabilizable

2. $\mathfrak{B}$ is set $S$-stabilizable

3. $\mathfrak{B}$ is $S$-stabilizable by interconnection.

Since for $n=1$ every controllable behavior is rectifiable, this theorem recovers the results obtained in [1], [2] and [3] for one-dimensional systems. The same happens with the corresponding property characterizations obtained in Propositions 2, 3 and 4.

\section{Concluding remarks}

An important issue is the characterization of each of the stabilizability properties in terms of behavior representations. Some results have already been obtained in [5] for set stabilizability, but, as already mentioned, a different notion of stability is used; also in [4] results on stabilizability by (non necessarily regular) interconnection were derived for the case of controllable behaviors.

For behaviors with rectifiable controllable part, we obtained a full characterization, that can easily be implemented in order to test stabilizability. However, the research reported in this paper did not go more deeply into this issue, as it was 
originally motivated by other aspects, more focussed on the establishment of relationships among the different stabilizability properties.

A natural question to ask is whether the conclusions presented in this paper carry on to the case of behaviors defined over $\mathbb{R}^{n}$. This is a nontrivial matter. Indeed, the results obtained here are strongly based on the finite dimensionality of discrete $n \mathrm{D}$ kernel behaviors that are stable with respect to some stability cone. This is in turn a consequence of (among others) the fact that the restrictions of an $n \mathrm{D}$ kernel behavior over $\mathbb{Z}^{n}$ to each of the $n$ axes in $\mathbb{Z}^{n}$ are $1 \mathrm{D}$ kernel behaviors over $\mathbb{Z}$. To our knowledge, no version of this result has been published in the literature for behaviors over $\mathbb{R}^{n}$. However, in case this property carries on to the continuousdomain case, it is clear that the same will happen with our results.

\section{References}

[1] J.C. Willems, On interconnections, control, and feedback, IEEE Transactions on Automatic Control Volume 42, pp. 326-339, 1997.

[2] P. Rocha and J. Wood, Trajectory control and interconnection of $1 \mathrm{D}$ and $\mathrm{nD}$ systems Rocha, SIAM Journal on Control and Optimization, Vol. 40, nr. 1, pp. 107-34, 2001.

[3] M. N. Belur and H. Trentelman, Stabilization, pole placement and regular implementability, IEEE Transactions on Automatic Control, Vol. 47, nr. 5, pp. $735-744,2002$. 
[4] H. K. Pillai and S. Shankar, A behavioral approach to control of distributed systems, SIAM Journal on Control and Optimization, Vol. 37, nr. 2, pp. 388408, 1998.

[5] M.E. Valcher, Stabilizability properties of two-dimensional behaviors, in Mathematical Theory of Networks and Systems, Proceedings of the MTNS'98 Symposium, Padova, July 1998, A. Beghi, L. Finesso and G. Picci, eds, Il Poligrafo, Padova, Italy, pp. 417-420, 1998.

[6] J.C. Willems, Models for Dynamics, Dynamics Reported, Vol. 2, 171-269, 1989.

[7] E. Zerz and V. Lomadze, A constructive solution to interconnection and decomposition problems SIAM Journal on Control and Optimization, Vol. 40, nr. 4, pp. 1072-1086, 2001.

[8] E. Zerz, Topics in Multidimensional Linear Systems Theory, Springer Lecture Notes in Control and Information Sciences 256, Springer, London, 2000.

[9] U. Oberst, Multidimensional constant linear systems, Acta Applicandae Mathematicae, Volume 20, pp. 1-175, 1990.

[10] J. Komornik, P. Rocha and J.C. Willems, Closed subspaces, polynomial operators in the shift and ARMA representations, Applied Mathematics Letters, Volume 4, nr. 3, pp. 15-19, 1991. 